\title{
Measurements of Phase Stability Over a Low-Level Tropospheric Path
}

\author{
M. C. Thompson, Jr., and H. B. Janes
}

(February 19, 1959)

\begin{abstract}
A knowledge of the statistics of atmosphere-induced variations in the phase of the received signal (i.e., variations in electrical path length) is essential in evaluating the reliability of any system using radio waves for measuring distance and/or velocity. This paper describes an analysis of phase variations measured at 9,400 megacycles per second over a 9.4mile path near Boulder, Colorado, during a 40-hour period in September 1958. The power spectral density of these variations is shown to be approximately proportional to $f^{-2.8}$ over a wide range of frequencies throughout the period of recording. The long-term phase variations are closely correlated with atmospheric refractivity measurements made at the path terminals.
\end{abstract}

\section{Introduction}

The National Bureau of Standards has been conducting a series of experiments to study the instability of the phase of vhf, uhf, and microwave radio signals transmitted over line-of-sight paths. ${ }^{1} \quad$ These experiments have included measurements of phase stability over "slanted" paths such as the Pikes Peak to Garden of the Gods path near Colorado Springs, Colo., and the Haleakala summit to Puunene path on the island of Maui, T.H. This paper describes some measurements of phase stability made at 9,400 Mc over a 9.4-mile path from Green Mountain mesa to Table mesa near Boulder, Colo. This path differs from the ones mentioned above in that both terminals are at approximately the same altitude. The data consist of: (1) $40 \mathrm{hr}$ of continuous recording of longterm variations in the phase of the radio signal; $(2)$ $40 \mathrm{hr}$ of continuous atmospheric temperature, pressure and relative humidity recordings; and (3) 21 samples (each approximately $5 \mathrm{~min}$ long) of shortterm phase variations. The analysis of these data consists of: (1) Estimation of the power density spectrum of the phase variations, using both longterm and short-term recordings; (2) estimation of the power density spectrum of the corresponding variations in radiofrequency of the received signal; and (3) correlation of the long-term variations in electrical path length (from the phase records) and surface refractivity (from the meteorological records).

\section{Path Terrain and Weather}

Figure 1 shows the location of the propagation path. It extends from Green Mountain mesa about $1 / 2$ mile west of the NBS, Boulder Laboratories, to Table mesa about 9 miles north of the Boulder Laboratories. The path length is 9.4 miles. As shown in figure 2 , each antenna was located at the edge of a steep slope to avoid ground reflections in the

1 J. W. Herbstreit and M. C. Thompson, Jr., Measurements 0 ! the phase of radio waves received over $t i$ ansmission paths with electrical lengths varying as a result of atmospheric turbulence, Proc. IRE 43, 1391 (1955). immediate vicinity. The path passed over two valleys which slope downward from west to east and are separated by a relatively flat mesa. This mesa contains a small lake (Roberts Lake), and appears to constitute the major ground-reflection area.

The measurements were made during the period 1100 , September 15, to 1625, September 17, 1958 .

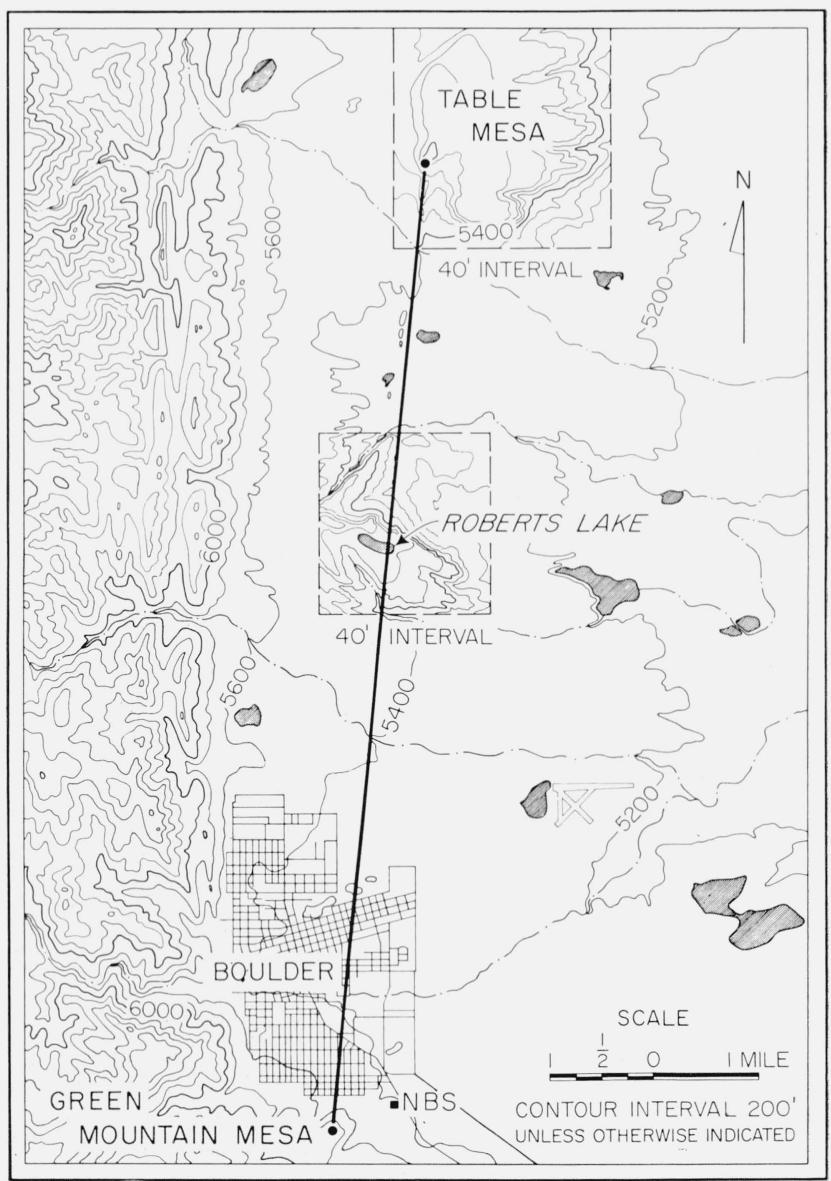

Figure 1. Green Mountain mesa-Table mesa path. 


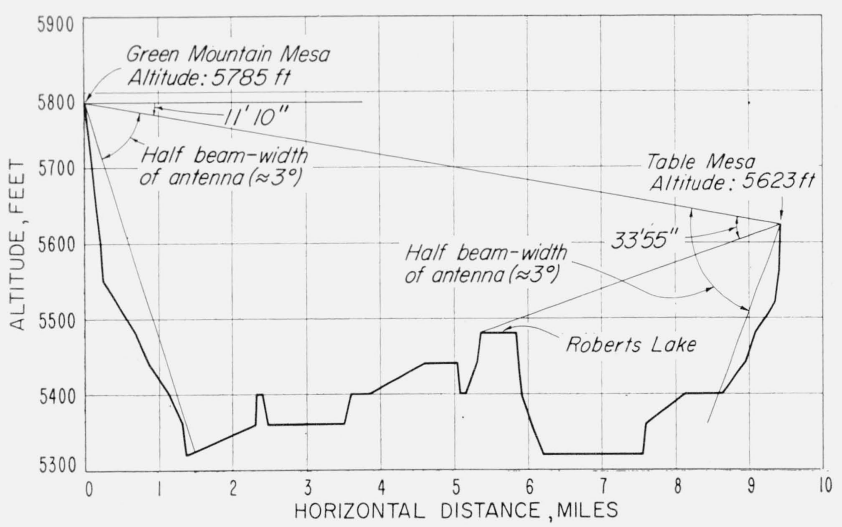

Figure 2. Terrain profile of Green Mountain mesaTable mesa path.

Weather conditions over the path during this period are summarized graphically in figures 3 and 4 , which show the wind velocity, barometric pressure, temperature, and relative humidity observed at the path terminals, and in the notes on figure 5 . A cold front moved across the path in a direction approximately normal to the path during the period 1000 to 1200 on September 15. The winds, temperature drop, and increase in relative humidity are indicated by figures 3 and 4. From about 1300 until 1830 the sky was overcast in all directions and a light rain fell steadily over all or part of the path, amounting to about .17 in. total accumulation. During the night patches of ground fog developed. As daylight came on September 16, from the tops of the mesas (the path terminals) fog could be seen for many miles to the northeast, east, and southeast, lying in the low areas of the terrain. Following sunrise at about 0540, these pockets of fog gradually disappeared until by 1000 the entire area was clear, with excellent visibility and partially cloudy sky. Pikes Peak, at a distance of 90 miles, was clearly visible from the Table mesa site from the onset of daylight. The remainder of the run was conducted in completely clear weather conditions.

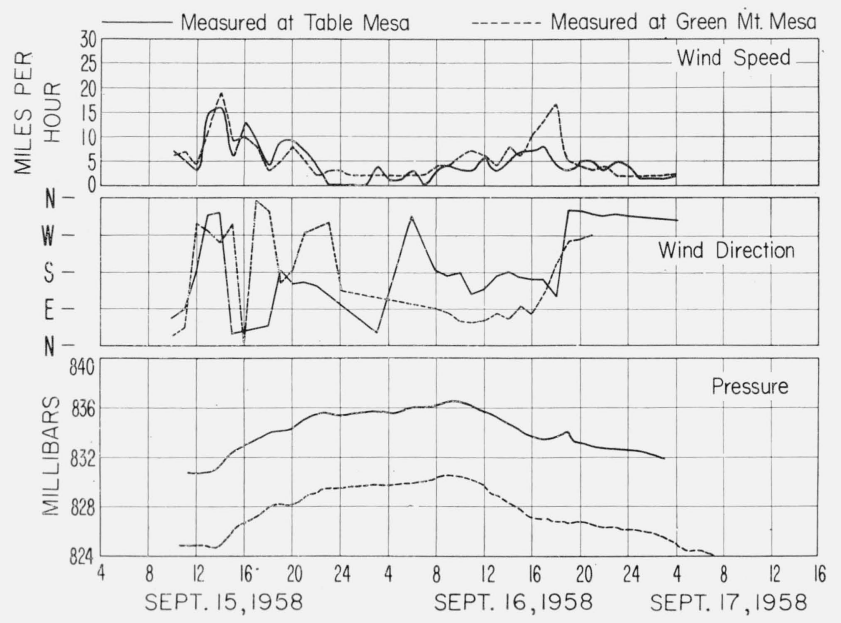

FIGURE 3. Summary of phase and meteorological data.

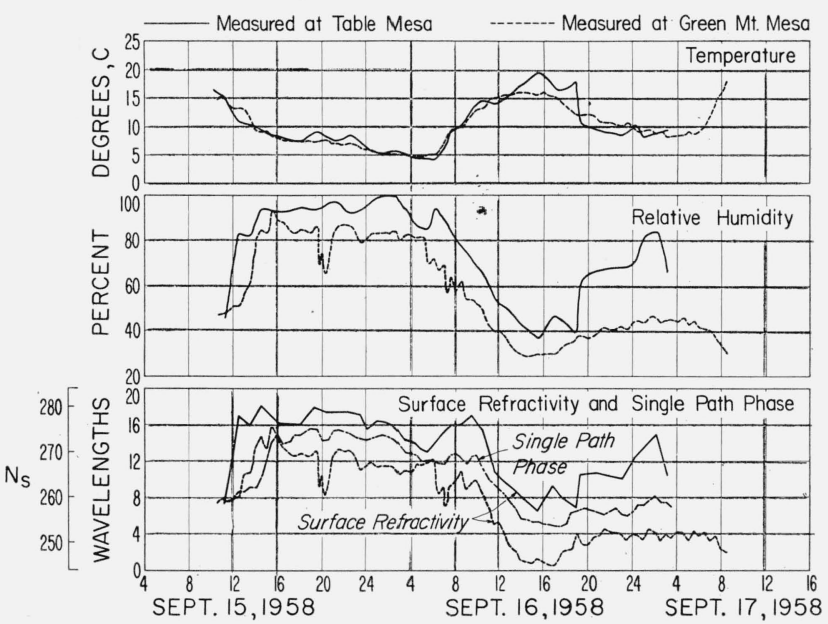

F. Figure 4. Summary of phase and meteorological data.

\section{Phase Measurement System}

The phase variations were measured by a system described earlier. ${ }^{2}$ It consists essentially of a quasisymmetrical arrangement in which two signals are compared after having traversed the same path in opposite directions. The measuring system employed had a noise level of the order of $0.1 \mathrm{deg}$ of phase due to combined thermal noise and electronic instabilities. At the operating wave length of about 1 in., this uncertainty corresponds to a physical displacement of either antenna (along the line of the path) of about $350 \mu$ in. Thus, the need for rigid antenna supports is apparent, and solid concrete piers $2 \mathrm{ft}$ in diam were used as mounts for the 18 in. parabolic dishes. The antennas were from 2 to $5 \mathrm{ft}$ above the immediate ground level. The frequency of the microwave measuring signal was stabilized to a few parts in $10^{8}$ using a phase-lock technique. ${ }^{2}$

The actual phase comparison was performed by a commercial electronic phase meter in conjunction with an electromechanical instrument developed at the Boulder Laboratories.

Two recording circuits were used to measure the phase variations in order to study the power spectrum over as wide a range of frequencies as possible. The output of one, referred to as the low-pass recording, consisted of a Varian chart moving at the rate of $0.1 \mathrm{ipm}$. A full-scale sweep of the pen represented a change of $1,800 \mathrm{deg}$ in the difference between the phase of the signals received at the twopath terminals (almost) simultaneously. The "single-path phase" data discussed here was obtained by simply dividing this "two-way phase" by two. The frequency response characteristic of this recording circuit is essentially flat up to about $1 \mathrm{cps}$. However, since the primary purpose of this record was to measure gross changes in phase, the dynamic range and chart speed used necessarily precluded the study of spectral frequencies above about $1 \mathrm{cpm}$. It was

${ }^{2}$ M. C. Thompson, Jr., M. J. Vetter, Single path phase measuring system for three-centimeter radio waves, Rev. Sci. Instr. (1959). 


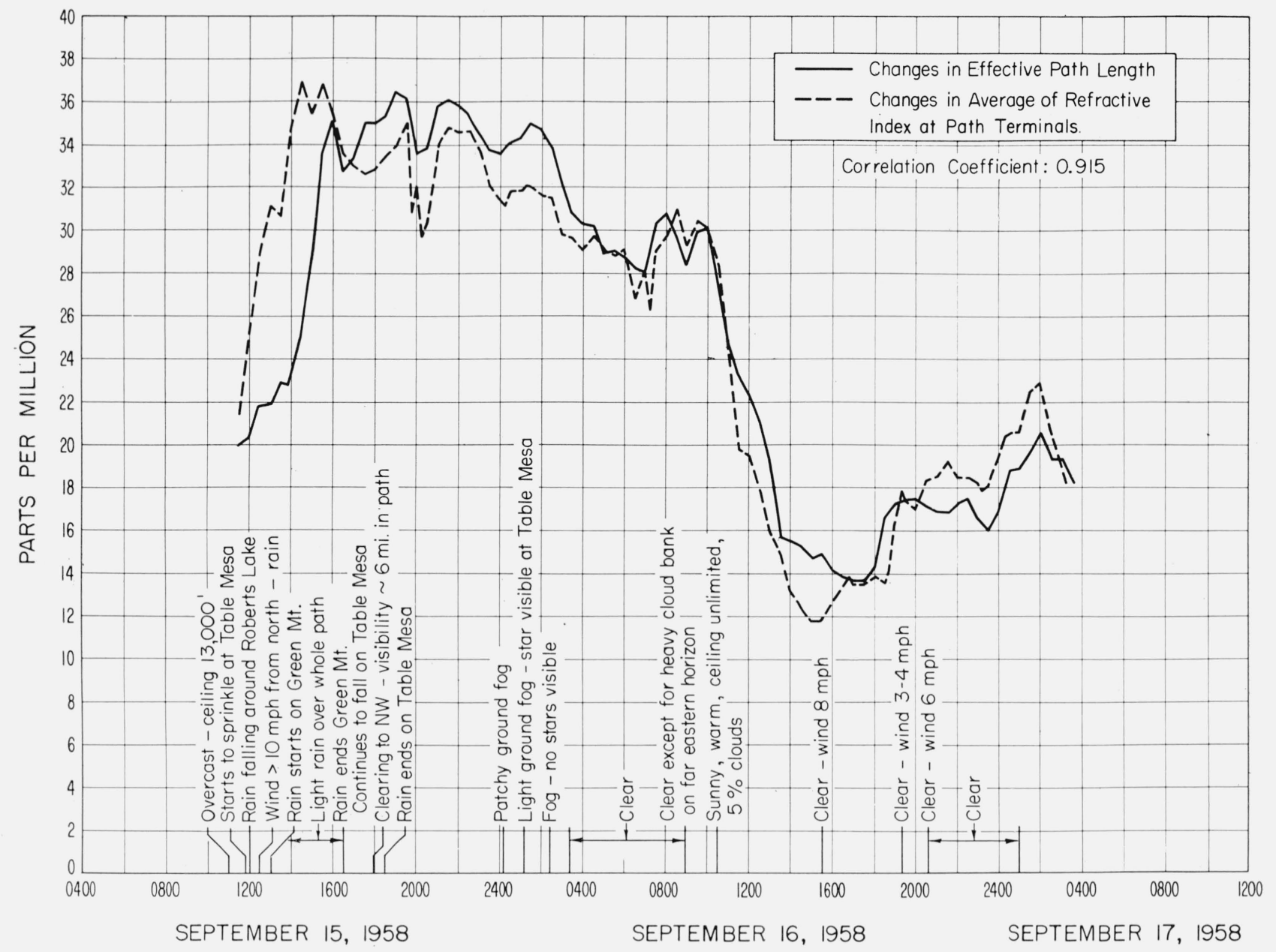

FJGURE 5. Comparison of variations in effective path length with average of surface refractive index at path terminals.

possible to record a wide range of values without loss of continuity because of an automatic scale-shifting arrangement which prevented the trace from going off scale.

The output of the second phase recording circuit, referred to as the band-pass recording, consisted of a Sanborn chart having full-scale ranges of 1.6 to 8 single-path deg and moving at the rate of about 60 ipm. Its frequency response characteristic consisted of a pass-band extending from about 0.03 cps to about $30 \mathrm{cps}$. This circuit was operated for 21 periods, each about $5 \mathrm{~min}$ in length, distributed over the 40 -hr period.

\section{Power Spectrum of Phase Variations}

One of the principal objectives of the experiment was to obtain information on the power spectrum of time variations in the phase of a microwave signal propagated over a line-of-sight path. The power spectrum might better be considered as the variance density spectrum, since it shows graphically how much of the total variance (mean squared deviations from the sample mean) was contributed by variations occurring in each of several narrow frequency bands distributed over the pass-band of the recording equipment. The method of estimating the power spectrum used here is that given by Blackman and Tukey. ${ }^{3}$ Briefly, it consists of computing the Fourier cosine transform of the autocovariance function and applying appropriate weighting functions to the result.

Seven of the twenty-one Sanborn recording periods were chosen for the power spectrum analysis, the only criterion used in their selection was that they be fairly evenly spaced over the $40-\mathrm{hr}$ period. The analysis of each period consisted of first computing the spectrum in the range 0.83 to $12.5 \mathrm{cps}$ for two adjacent data segments each $0.5 \mathrm{~min}$ in length, using a sampling interval of 0.04 sec. Then the two segments were combined, the sampling interval increased to $0.2 \mathrm{sec}$ and the spectrum computed for the range 0.083 to 2.5 cps. This yielded 14 sample spectra in the higher frequency range and 7 in the lower range of the Sanborn records.

R. B. Blackman and J. W. Tukey, The measurement of power spectra from the point of view of communication engineering, Bell System Tech. J., Pts. 1, 2 , $37,185,485$ (1958). 
From consideration of the frequency response of the recording circuit, it is felt that the seven lower range spectra are not seriously contaminated by frequencies below $0.083 \mathrm{cps}$. However, the data for the 14 higher range spectra were subjected to a digital trend removal process, ${ }^{4}$ since they would otherwise be contaminated by variance contributed at frequencies beiween the lowest spectral frequency estimated $(0.83 \mathrm{cps})$ and the lower limit of the pass-band (about $0.03 \mathrm{cps}$ ). (In all cases, the spectra were corrected to allow for the recording circuit response.)

${ }_{4}$ This process consists simply of computing the deviations of the variable from a running average, computing the power spectrum of these deviations, and cora running average, computing the power spectrum of these deviations, and cor-
recting the result for the frequency response of this high-pass "filter." This, in rectect, filters out variations at spectral frequencies below a cutoff frequency which is determined by the averaging time.
The analysis of the Varian low-pass data consisted of first digitizing the entire 40-hr run with a sampling interval of $0.5 \mathrm{~min}$. Then five sample spectra were computed, each using 6 to $8 \mathrm{hr}$ of data, covering the range from 0.00111 to $0.0167 \mathrm{cps}$, ( $1 / 15$ to $1 \mathrm{cpm})$. Then two sample spectra were computed, each using $15 \mathrm{hr}$ of data and covering the range from 0.000278 to $0.00417 \mathrm{cps}$ ( $1 / 60$ to $1 / 4 \mathrm{cpm}$ ), and finally, one spectrum utilizing all $40 \mathrm{hr}$ of data and covering the frequencies from 0.0000926 to $0.00139 \mathrm{cps}(8$ cycles per day to $1 / 12 \mathrm{cpm})$. In each case, the data were subjected to the trend removal process to minimize the effects of variations at frequencies below the range covered in the computation.

The individual spectral densities for each sample are plotted in figure 6 . The points shown as open

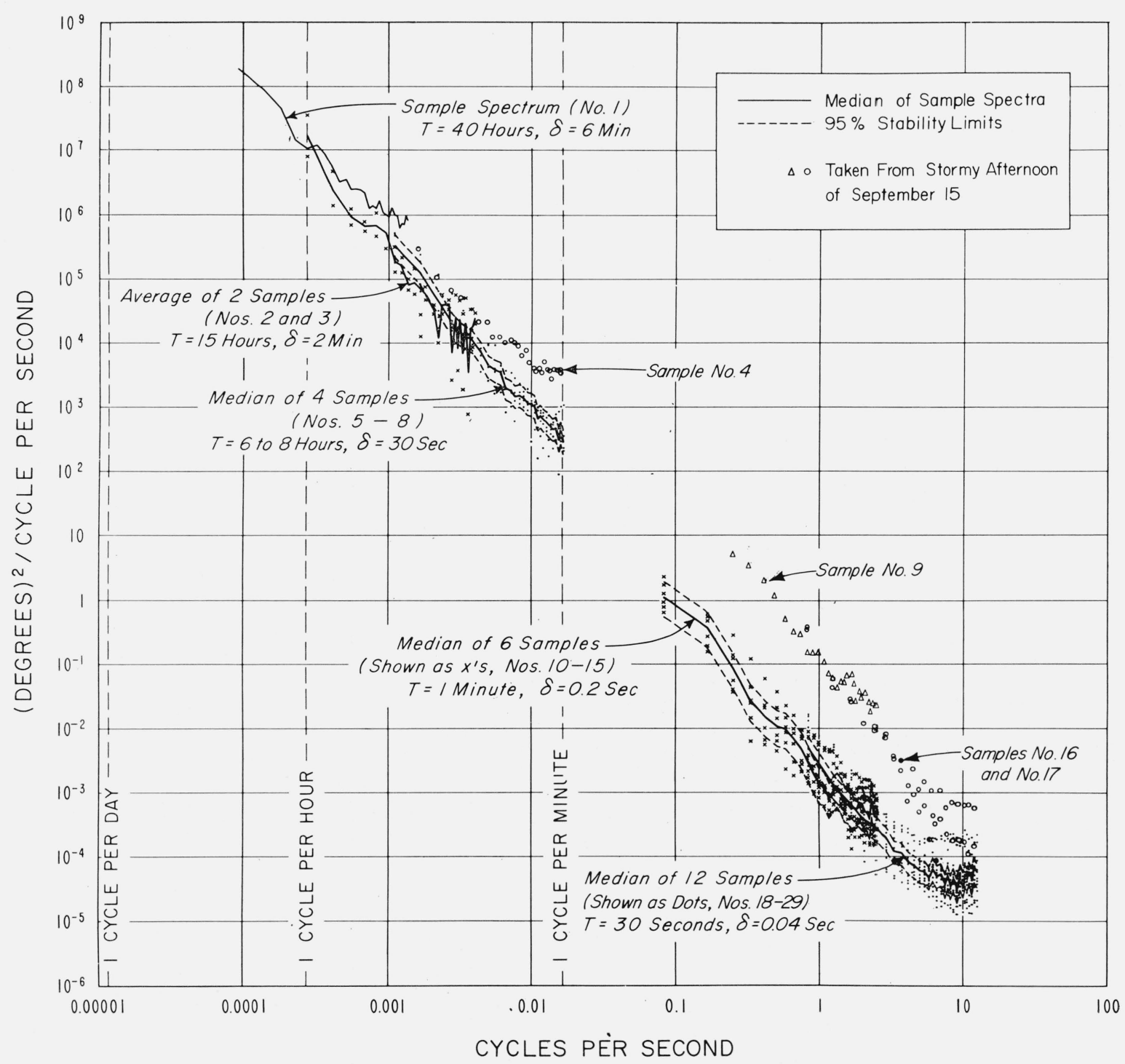

Figure 6. Power spectrum of phase variations in 9,400 Mc transmissions over a 9.4-mile path. 
dots were computed from data recorded during the stormy afternoon of September 15 . The phase recording during that period was characterized by rapid fluctuations which did not recur during the remainder of the experiment. The corresponding spectra were sufficiently different from all the subsequent ones to warrant distinguishing them from the rest. The solid lines join the medians of the sample spectral densities for each group of spectra, excluding those shown as open dots. The range of the points at any given frequency is determined both by sampling fluctuations and by more or less long-term changes in propagation conditions. Some measure of the relative magnitude of these two effects can be obtained by considering the distribution of spectral densities to be expected if the samples had been taken from the same statistical population, i.e., if the time variations in phase constituted a stationary process. In that case the spectral density estimates at each frequency would be distributed according to the chisquared distribution with an effective number of degrees of freedom determined by the number of data points used in each estimate; the number of frequencies for which estimates were computed; and, to a very limited extent, by the slope of the spectrum. Using the sample median to approximate the "true" median, the dashed lines labelled "95 percent stability limits" represent the bounds within which 95 percent of all such spectral estimates might be expected to fall if the process were indeed stationary. In view of the large long-term changes in phase and meteorological conditions shown in figures 3 and 4 , it is not surprising that about 50 percent of the spectral estimates fall outside the 95 percent stability limits, indicating systematic changes in spectral density over the 40-hr period. In fact, it is remarkable that the sample spectra are so similar, both in slope and magnitude.

It will be noted that there is a tendency for the slope to approach zero at the high frequency end of each set of spectra. This is caused by "aliasing", or the addition to the computed densities of power actually present at the higher frequencies For spectra having steep slopes (such as these) aliasing causes an error which is about two to one at the highest frequency of each spectrum and which diminishes rapidly with frequency. Additional flattening of the spectrum above about $6 \mathrm{cps}$ is probably caused by "noise" introduced in the process of reading and analyzing the data, and should be ignored. Making allowances for aliasing and reading error noise, it appears that the slope of the phase spectrum is essentially constant over the range from 8 cycles per day to $12.5 \mathrm{cps}$, the power density being approximately proportional to $f^{-2.8}$.

Figures 7 and 8 show the seven spectra from the lower frequency range of the Sanborn data and the five spectra from the high-frequency range of the Varian data identified individually by time of day. In general, there appears to be no outstanding correlation between these spectra and either the time of day or gross meteorological variations (with the obvious exception of the spectra taken from the

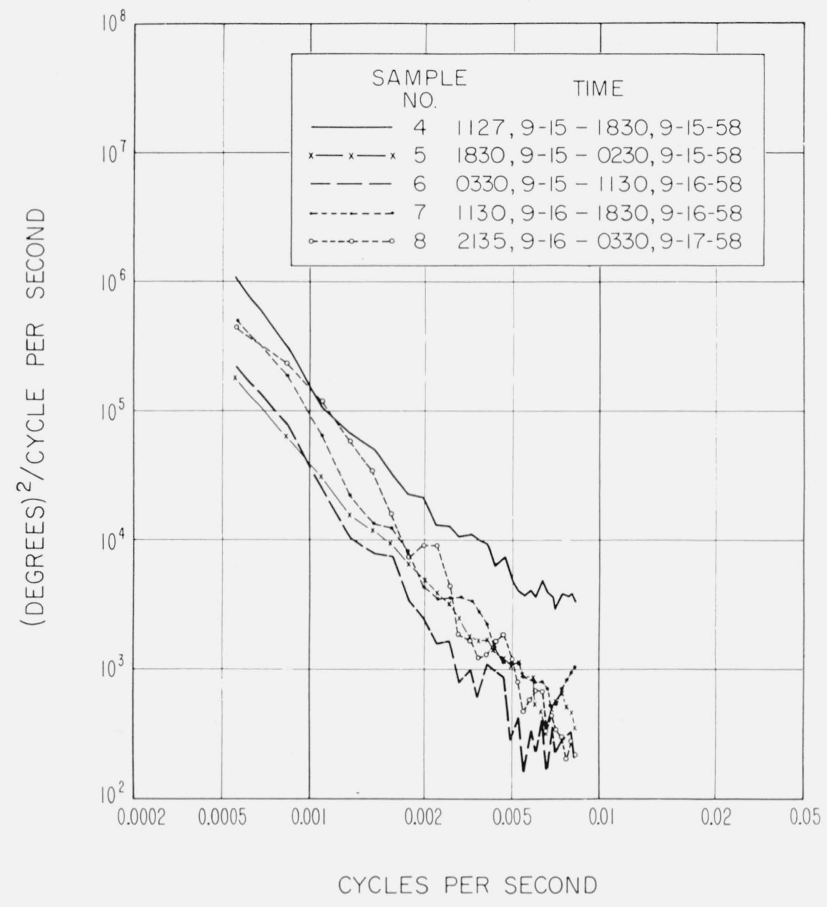

Figure 7. Sample power spectra of phase variations in 9,400 Mc transmissions over a 9.4-mile path.

Low-pass Varian recording.

stormy afternoon of September 15). One is tempted to attach some significance to the fact that the phase variance was somewhat lower during the period of high humidity following the storm than it was later during the period of warmer and drier weather. This is, of course, too small a data sample to warrant drawing any conclusions, and the correlation of phase and meteorological data will be more thoroughly studied in subsequent measurements.

During the afternoon of September 17 (after the 40-hr run), the polarization of the antennas at both ends of the path was changed from horizontal to vertical and back again to horizontal. A spectrum analysis of samples of phase variations before and after the changes indicated that polarization has no apparent effect on the phase spectrum. This lends some support to the belief that there is little possibility of multipath effects caused by ground reflections from the mesa at Roberts Lake (see figs. 1 and 2).

In summary, the phase spectrum analysis described here indicates that: (1) The slope of the power spectrum is nearly constant over the range of frequencies from $0.0001 \mathrm{cps}$ to about $10 \mathrm{cps}$, the spectral densities being roughly proportional to $f^{-2.8}$; (2) except for the afternoon of September 15 when a rainstorm crossed the path and caused large high frequency fluctuations in phase, the power spectrum remained fairly constant as a function of time, both with regard to magnitude and to slope; and (3) the phase variations appear to be insensitive to changes in polarization. 


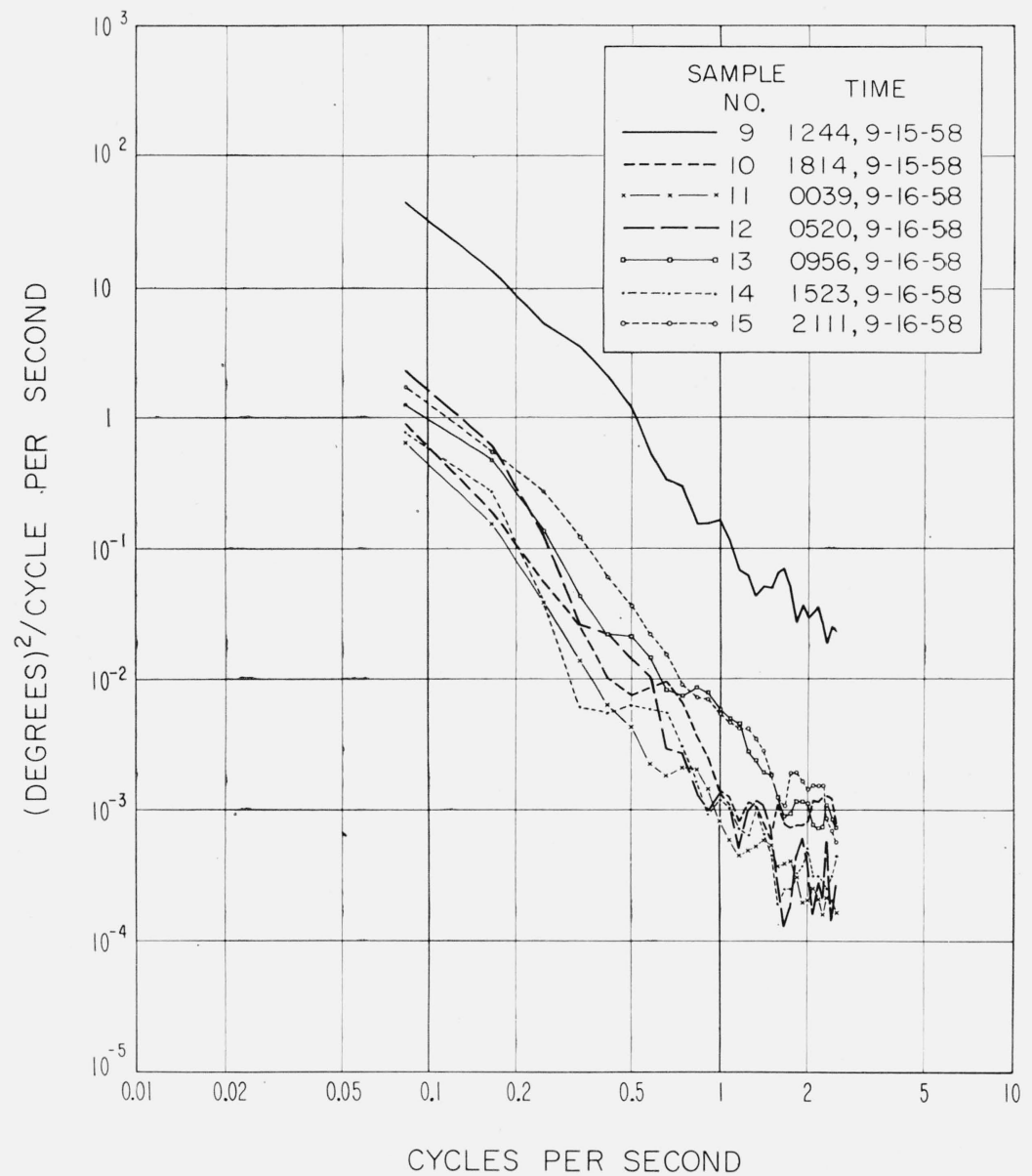

FIGURE 8. Sample power spectra of phase variations in 9,400 Mc transmissions over a 9.4-mile path.

Band-pass Sanborn recording

\section{Power Spectrum of Radiofrequency Deviations}

The time variations in phase can be interpreted as deviations in the radiofrequency of the received signal, and a simple relationship exists between the power spectra of these two parameters. To express the effects of the atmospheric turbulence on the propagated signal in terms of its frequency-modulation effects rather than phase variations, we may write the transmitted and received waves (ignoring attenuation) as follows:

$$
\begin{aligned}
& e_{T}=\epsilon^{i \omega t} \\
& e_{R}=\epsilon^{i(\omega t-k l)}=\epsilon^{i(\omega t-\theta)} .
\end{aligned}
$$

If we define the frequency of the received signal as the time derivative of the total phase term we obtain:

$$
\omega^{\prime}=\omega-\frac{\partial \theta}{\partial t}
$$

or

$$
\Delta \omega=\omega-\omega^{\prime}=\frac{\partial \theta}{\partial t}
$$

Thus, the frequency deviation due to the turbulence is proportional to the slope of the phase records obtained in the experiment.

If $\theta$ is some function of time which can be represented by the Fourier series:

$\theta(t)=a_{0}+a_{1} \cos 2 \pi f_{1} t+b_{1} \sin 2 \pi f_{1} t+\ldots+a_{n} \cos$

$$
2 \pi f_{n} t+b_{n} \sin 2 \pi f_{n} t+\ldots .
$$

its power spectral density at the spectral frequency $f_{n}$ is proportional to $\left(a_{n}^{2}+b_{n}^{2}\right)$. Similarly, the frequency deviations can be represented by tio series:

$$
\begin{aligned}
\frac{\partial \theta}{\partial t}=-2 \pi f_{1} a_{1} \sin 2 \pi f_{1} t+2 \pi f_{1} b_{1} \cos 2 \pi f_{1} t+\ldots \\
\quad-2 \pi f_{n} a_{n} \sin 2 \pi f_{n} t+2 \pi f_{n} b_{n} \cos 2 \pi f_{n} t+\ldots,
\end{aligned}
$$

and its spectral density at $f_{n}$ is proportional to $\left(2 \pi f_{n}\right)^{2}\left(a_{n}^{2}+b_{n}^{2}\right)$.

Figure 9 shows the frequency variation spectra corresponding to the phase spectra shown in figure 6 . They were obtained simply by multiplying the 


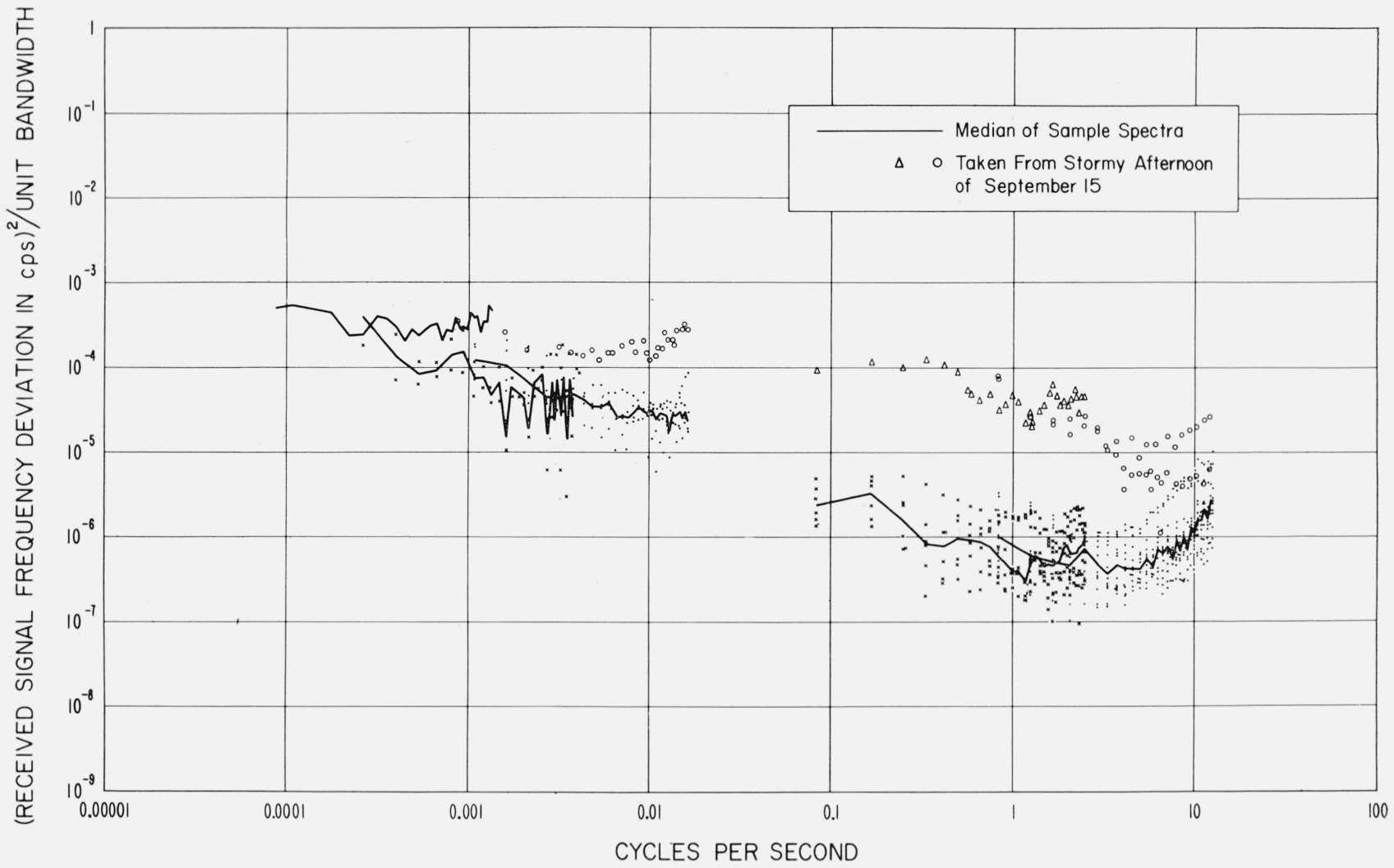

Figure 9. Power spectrum of radiofrequency deviations in 9,400 Mc transmissions over a 9.4-mile path.

phase spectral density at the frequency $f$ cycles per second by $\left(\frac{\pi f}{180}\right)^{2}$. The spectral density of radiofrequency variations decreases relatively slowly with increasing spectral frequency, being approximately proportional to $f^{-0.8}$. (The exponent here is simply the value obtained for the phase spectrum increased by two.) The apparent increase in power at the high frequency end (above about $6 \mathrm{cps}$ ) is caused by the reading error noise mentioned previously and should be ignored.

\section{Comparison of Phase and Atmospheric Refractivity Variations}

Variations in the phase of the received signal represent variations in the electrical length of the propagation path, which is proportional to the atmospheric refractivity integrated along the path. Hence, the long-term changes in refractivity measured at a point on the path should be correlated with the long-term changes in electrical path length as deduced from the phase records. This correlation is illustrated in figure 4 , in which the refractivity values computed from temperature, pressure, and humidity recordings at each terminal are plotted with the long-term phase variations read from the Varian record. It is perhaps more clearly shown in figure 5, in which time variations in the average of the refractive index at the path terminals and the changes in electrical path length are both expressed in parts per million. This illustrates the fact that the two variables are not only closely correlated (correlation coefficient: 0.915), but their fluctuations agree in magnitude as well.

The following personnel participated in the experiment reported here: J. T. Collins, F. E. Freethey, W. B. Grant, D. P. Harris, H. B. Janes, A. W. Kirkpatrick, D. Smith, M. J. Vetter, and D. M. Waters. Project leader was Moody C. Thompson, Jr.

The assistance of J. W. Herbstreit and K. O. Hornberg is also gratefully acknowledged.

Boulder, Colo. (Paper 63D1-7). 